\title{
Knowledge translation in obstetrics and gynaecology: a pilot study of physical and online media
}

\begin{abstract}
Background: Knowledge translation (KT) is defined as the exchange, synthesis and ethically sound application of knowledge, and is critical for translating research findings into the public's hands. In obstetrics and gynaecology, there are only a few poignant examples of strategic knowledge translation. The aim of this project was to pilot test a combination of face-to-face as well as online KT strategies for the purpose of disseminating scientific findings in obstetrics and gynaecology to a public audience.
\end{abstract}

Methods: We delivered a series of face-to-face public forums, and online YouTube videos and a webinar in which experts in obstetrics and gynaecology delivered key findings in research.

Results: Participants to face-to-face events were more likely to have a personal interest in the topic, that these events are an opportunity to correct health-related myths, and that the interactive nature of the forum allowed women to have their individual questions addressed.

Interpretation: In terms of online KT, websites can be an effective and inexpensive way of having a broad reach, that Facebook is an effective way to interact with young, female members of the public, and that Webinars are an inexpensive means of doing KT. A number of lessons were learned that will assist in future strategic delivery of KT in the area of ob/ gyn.

Keywords: Knowledge translation, Public forum, Online education
Volume 5 Issue 2 - 2016

\author{
Lori A Brotto,' Christine Clark, ${ }^{2}$ Roxana \\ Geoffrion,' Deborah Money,' Leslie \\ Sadownik,' Kelly B Smith,' Paul Yong,' \\ Geoffrey Cundiff' \\ 'Department of Obstetrics and Gynaecology, University of \\ British Columbia, Canada \\ Independent Contractor, Canada
}

Correspondence: Lori A Brotto, Department of Obstetrics and Gynaecology, University of British Columbia, Vancouver, BC, Canada,Email lori.brotto@vch.ca

Received: September 09, 2016 | Published: September 29, 2016

\section{Introduction}

"Knowledge translation (KT) is defined as the exchange, synthesis and ethically sound application of knowledge-within a complex system of interactions among researchers and users - to accelerate the capture of the benefits of research... through improved health, more effective services and products, and a strengthened health care system"

KT is increasingly being used and encouraged in health sciences due to the recognition that many discoveries in science either do not translate or take decades to translate into changes in practice or policy, but remain buried in academic journals that are not readily accessed. There are three main recipients of KT: practitioners, policy makers and the general public. Traditional models of KT consider the researcher as "expert", whereas newer models encourage collaboration between researcher, policy maker, and public. ${ }^{2}$ Translating knowledge to the public first involves clarifying the content for the intended audience. Creating content which is accessible is a high priority given that the typical outlets for new scientific discoveries-academic journalsare not readily accessible by the public. ${ }^{3}$

One of the strategic initiatives of the Canadian Institutes of Health Research (CIHR) is KT to a public audience. Only a small proportion of scientific discoveries are disseminated directly to the public. ${ }^{4}$ with the vast majority of scientific discoveries not making their way to the public or stakeholders who not only have a vested interest in the findings, but often support the federally-funded research agencies that such research relies on. A survey of social science researchers in Canada found a large gap in translating research to application; $16 \%$ of 1229 respondents stated that their research results usually or always fostered clinical applications whereas $56 \%$ reported that their research never (including does not apply) or rarely lead to such practical applications. ${ }^{5}$

Strategies for KT rest upon a combination of passive and active approaches including training workshops, professional development opportunities, communication through print and electronic media and personal, and face-to-face contact. ${ }^{6}$ Online media is a particularly effective KT strategy.?

\section{Knowledge Translation in Obstetrics and Gynaecology}

Relative to other areas of medicine, KT is not universally adopted across all domains of obstetrics and gynaecology. One exception is in the area of pregnancy: three quarters of surveyed fellows in Australia and New Zealand reported that pregnancy information from websites had been brought to their attention by patients, indicating that women are accessing the internet for their health information and are speaking with their doctors about it. ${ }^{8}$ A focus group study of pregnant women and young mothers in the USA reported that they used the Internet to confirm their health-related beliefs or reassure themselves that their perceptions were correct. Participants also expressed preference for online clinical health information that is presented by experts. ${ }^{9}$

The adoption of intrauterine contraception in the United States is low ( $1.3 \%$ annually) compared to developed European countries (France 20\%, Norway 24\%, Finland 26\%) despite overwhelming evidence of its effectiveness, low-cost, low user-error and safety as a long acting reversible-contraceptive method, and barriers in effective KT are thought to contribute to these disparities. A survey of 816 physicians and non-physicians servicing more than 100 female contraceptive patients per year found that providers were generally uninformed about the method or held long disproven beliefs about its safety and were unlikely to counsel their patients on this method. ${ }^{10}$ This creates a pivotal KT opportunity. 
One success story in the use of KT in gynaecology is the opportunistic salpingectomy initiative. In British Columbia, after findings were published that removal of the fallopian tubes in high- and averagerisk populations for ovarian cancer could prevent the spread of cancer cells, ${ }^{11}$ the Ovarian Cancer Research Program launched an educational campaign to all gynaecologists in $\mathrm{BC}^{12}$ using an educational DVD which recommended surgical removal of the fallopian tubes (bilateral salpingectomy) at the time of hysterectomy. Following the campaign, researchers found that uptake in British Columbia over 4 years was significant: hysterectomy with bilateral salpingectomy increased from $5 \%-35 \%$ and salpingectomies for sterilization increased from $0.5 \%-$ $33 \% .^{13}$ In Canada bilateral salpingectomy at time of hysterectomy increased to more than $11 \%$ in 2011 from less than $1 \%$ in $2006{ }^{12}$ In a 2013 survey of Canadian gynaecologists, most respondents were aware of risk-reducing salpingectomy as a possible method to prevent ovarian cancer in women at average risk. ${ }^{14}$ Despite the success of this initiative, however, many other areas of obstetrics and gynaecology have not capitalized on KT methods, and this directly impacts the health of women. Even the Society of Obstetricians and Gynaecologists of Canada (SOGC) do not yet have guidelines for education in KT. ${ }^{15}$

\section{"GYN in the know" KT strategy}

The goal of this project was to disseminate information about innovations in women's health to the public. There were two main streams of dissemination:

1. Face-to-face dissemination using public forums hosted by experts in specialized areas of gynaecology, and

2. Online dissemination using a combination of a web-based and social media campaign.

As this was a pilot project uniting many subspecialty areas in gynaecology, our aim was to explore effective versus ineffective means of disseminating science to the public.

\section{Methods}

\section{Face-to-Face Dissemination}

Five public forums were scheduled featuring topics that covered new information and innovations in gynaecology over a six-month period in 2014 (Table 1). Each forum lasted approximately an hour with 15-20 minutes of didactic presentations from the expert speakers and 40-45 minutes of an interactive component that included open questions from the audience. Before each forum a survey was given to each attendee that assessed the participant's prior knowledge on the forum's topic. Attendees' responses to the information presented were then tracked in a post-forum questionnaire. For example, true/false questions assessed baseline education about the particular condition discussed, and participants were asked what they would do with the information acquired. The forums were advertised through local newspaper ads, an informational website www.gynintheknow.com, and a social media campaign.

Table I Women's health topics covered during public forums

\begin{tabular}{ll}
\hline Date & Topic \\
\hline February 5, 2014 & Genital Pain and Sexual Health \\
\hline February 19, 2014 & $\begin{array}{l}\text { Urinary Incontinence and Prolapse } \\
\text { Pelvic Floor Health during Pregnancy and } \\
\text { March 12, 2014 }\end{array}$ \\
\hline
\end{tabular}

Table continued..

\begin{tabular}{ll}
\hline Date & Topic \\
\hline April 23, 2014 & Personalized Medicine for Women \\
May 26, 2014 & Endometriosis, what are the signs? \\
June 17, 2014 & $\begin{array}{l}\text { Hepatitis B, HIV and other infections, can my } \\
\text { baby get it? (webinar) }\end{array}$ \\
\hline
\end{tabular}

\section{Online Dissemination}

GYN in the know Website: A new website (www.gynintheknow. com) was created to provide information and resources relating to the topics covered in the face-to-face forums. The website was open access, and anyone could post questions which were then routed by our coordinator to the appropriate expert. Brief educational videos summarizing each of the forum topics were also housed on the website's homepage. Data were collected using Google Analytics and ranged from number of visits and new users to user behaviour and acquisition information.

Social Media: There was an active social media campaign throughout this project that utilized a combination of Facebook and Twitter to promote public events, build confidence in the GYN in the Know brand, and disseminate scientific findings relevant to the topics covered in the forums. Evidence of activity or engagement in the online presence was measured through tracking the number of clicks, impressions and interactions with each Facebook post, and the number of re-tweets for each twitter post. We also cross-referenced the social media activity with the number of visits to the website. In addition, we recorded demographic information about website visitors and Facebook fans.

YouTube Videos: Brief videos were created which ranged from 2-10 minutes in length, and summarized key information about women's health topics, and in some cases, tips for patients, based on the forum topics. Videos were disseminated via a GYN in the Know YouTube channel. We collected data using YouTube analytics on the demographics of the viewers and their viewing behaviour (how long, where the user dropped off, the device type used to view the video).

Webinar: One 47 minute long webinar was held live using Google Hangouts On Air on the topic of pregnancy and sexually transmitted infections called "Hepatitis B, HIV and other infections: Can my baby get it?". The webinar was viewed live and also saved to the YouTube channel so those who would like to view it later could view the entire video. We tracked the number of live viewers, later viewers, and their demographics, and viewing behaviour through YouTube analytics.

\section{Results}

\section{What worked?}

a. Women with a personal interest in the given health topic were motivated to attend in-person forums and share the information learned: A total of $50-80 \%$ of forum participants attending the talk focused on genital pain reported that they would speak to a care provider or partner about the information learned, thereby suggesting they personally experienced such pain. As well, a follow-up telephone call with a random sample of forum attendees revealed that $75 \%$ had followed through with their stated intention of sharing the information learned at the forum with someone.

b. Public forums are an opportunity to correct myths and disseminate accurate information about women's health to the public: We used true/false questions before the start of each forum to gather participants' baseline knowledge. On some items 
assessing baseline knowledge about the condition (e.g., "genital pain in women is rare"), women scored only $50 \%$ correctly, evidencing the need for accurate information dissemination, particularly among sensitive topics within women's health. In forums that provided specific information on skill building (e.g., the importance of practicing kegel exercises during pregnancy; the role of effective sexual stimuli in eliciting women's sexual response), most women indicated in their post-forum questionnaire that they were highly motivated to enact the skills taught during the forums.

c. The interactive nature of the forum allowed women to have their individual questions addressed: Given that two thirds of the scheduled forum time was devoted to interactive activities including a moderated question and answer period, this provided a unique opportunity for women to acquire new information directly from the health experts in the given topics.

d. People talk: Responses to the questions on the forums' surveys varied, however, one common denominator was that many women indicated that they would share the information they had learned with others (range $25 \%-63 \%$ of respondents). Encouraging public information sharing could bolster dissemination.

e. Creation of a website can be an effective and inexpensive way of disseminating KT to the public across a variety of countries: Table 2 indicates that we had individuals from 74 different countries interacting with our gynintheknow.com website. The total number of sessions (visits to the website) during the period of the project were 3,275 with 2,600 unique users.

Table 2 Data on how information was obtained about GYN in the know

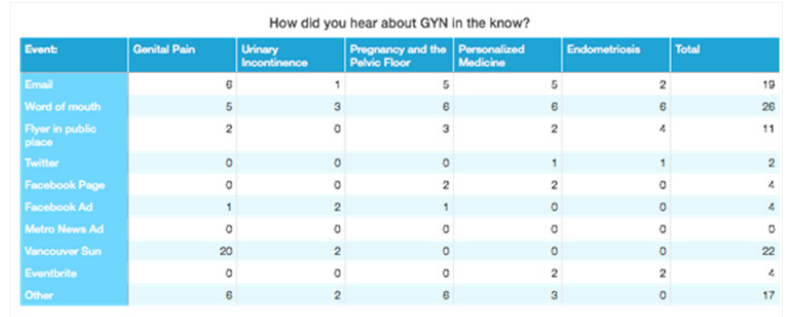

f. Search engine optimization (SEO) can help interested viewers find the website: Table 3 shows that $41.8 \%$ of the website viewers were directed to the site through search engine results. Good SEO increases the chances that the site will be found when users search for it. ${ }^{16}$ The GYN in the Know website was successful in terms of user acquisition. Most users were acquired through organic search, meaning that users found the website by searching for relevant terms in search engines like Google.com. Certain measures were taken to ensure search engine optimization including prominent use of relevant keywords in headings and throughout multiple pages of the website.

g. People are looking on the web for specific information: Ten emails were sent through the contact us page and inquired about various gynecological conditions and general questions such as seeking referral sources and information on specific clinics.

h. Facebook is an effective way to interact with young, female members of the public: Most of our Facebook visitors were women and over half of our Facebook visitors (56\%) were between the ages of 25-44. An analysis of Facebook likes and unlikes is an effective means of deciphering which types of posts/ which content areas are most relevant to a public audience. We had a total of two unlikes for the entire year-long duration of our Facebook presence, suggesting that the material presented was appropriate to the public.

i. Webinars are inexpensive means of KT: One of the known barriers in consistently incorporating KT relates to the high costs that are not often covered in research funding. ${ }^{17,18}$ We used Google Hangouts on Air and Logitech webcam technology to record our webinar. Google Hangouts on Air creates a live broadcast using YouTube which we embedded on our website. Over its lifespan, the webinar received 200 total views, suggesting that webinars might carry long-term value because of their ability to be accessible at a time convenient for viewers. Unfortunately, however, we were unable to collect any survey information from viewers, so webinars are excellent tools for reaching a large number of people but less useful when trying to collect data for ongoing analysis.

Table 3 User acquisition for gynintheknow.com

\begin{tabular}{lll}
\hline Channel & \# of sessions & \% of sessions \\
\hline Organic Search & 1,344 & $41 \%$ \\
Direct & 718 & $22 \%$ \\
Referral & 690 & $21 \%$ \\
Social & 524 & $16 \%$ \\
\hline
\end{tabular}

\section{Lessons learned}

1. Most attendees to our public forums learned about the event through feature pieces in newsprint: Considerable time and effort was spent publicizing the forums via social media yet our analysis revealed that most participants learned of the forum through a feature piece in one of the local newspapers, and secondly, from posted advertisements in specialty clinics that treat the given disease topic. This finding suggests that social media, alone, may not be the most effective way to alert the public to face-to-face forums.

2. Venues matter: Our face-to-face forums took place in a downtown university campus which was difficult to access by vehicle. This venue may have prohibited more of the public from attending. Moreover, the timing of the forums may have been too late as they occurred in the evening and did not coincide with typical work end hours.

3. Audience members are savvy: The knowledge assessment portion of the surveys revealed that audience knowledge base on each topic varied greatly. During our telephone follow ups, audience response on how much of the information was new showed that many felt they had not learned anything new (Figure $1)$.

4. Most viewers remain on a website or video for only 1-2 minutes: For videos that were considerably longer, we saw a dropping off of viewers, suggesting that the most important information to be disseminated to the public must be presented within the first 1-2 minutes. Google Analytics cannot track the number of seconds on the last page visited, therefore sessions which only included one page view are counted as $0-10$ seconds. ${ }^{16,18}$ We found a slightly longer duration of viewing when our videos were posted on YouTube (1:01-5:59 minutes) suggesting the YouTube might be a better way of disseminating video information. Most individuals who reached both our website and YouTube videos did so through an organic search. 
Given that individuals' first impression guides behaviour, ${ }^{19}$ this suggests that effective strategies to capture viewers must take place within the first few seconds of any online KT method.

\begin{tabular}{|c|c|c|c|c|c|c|c|c|}
\hline \multicolumn{9}{|c|}{ How much of the intomation you leamed was new? } \\
\hline WHOW & NWFW & WW & & WWWW & 10\%11\% & OWW & Mo Promonom & \\
\hline & 2 & 2 & 3 & & 1 & 2 & 4 & 2 \\
\hline \multicolumn{9}{|c|}{ - Number of respondents } \\
\hline 3 & & & & & & & & \\
\hline 2 & & & & & & & & \\
\hline 1 & & & & & & & & \\
\hline 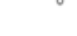 & 80\%-89\% & 70\%-79\% & 50\%-59\% & $20 \% \cdot 29 \%$ & $10 \% \cdot 19 \%$ & $0 \%-9 \%$ & No Response & \\
\hline
\end{tabular}

Figure I New information acquired during public forums.

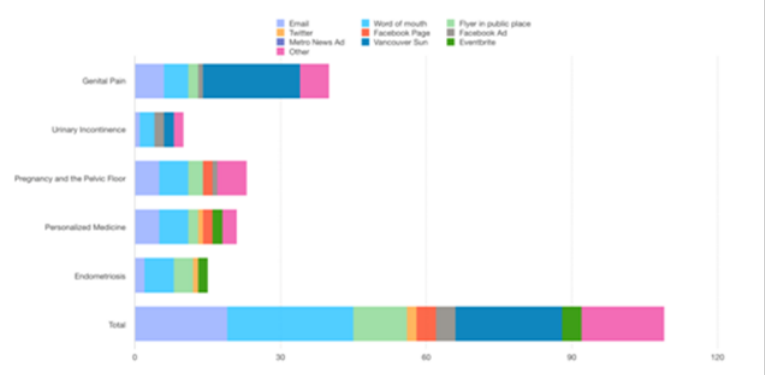

Figure 2 Data on how information was obtained about GYN in the know.

A homepage must be appealing: An analysis of our page views for www.gynintheknow.com revealed that viewers most often viewed the homepage, and not the events or resources page. This finding suggests that the most important information to be disseminated should appear on the main (home) page and not require additional clicks or navigation. Best practices in web design state that any calls to action (e.g., "contact us", "join our mailing list", "come to a forum") should appear in large text and the user should not have to scroll down to see them. ${ }^{20}$

Online vehicles may be limited in their reach to older women: An analysis of our Facebook visitors indicated that only $4 \%$ were aged 65 years or older. This result suggests that, for certain health topics that pertain to an older age group, Facebook may not be the most effective KT strategy.

Facebook ads are a better tool for online-only engagement. Only 4 of our forum attendees had learned about the event through Facebook Ads. However, we found that engagement on Facebook reached up to 1,700 people suggesting that broader online-only topics can be disseminated through Facebook and possibly other social media.

\section{Interpretation}

A number of conclusions can be drawn with regards to face-toface public events regarding knowledge translation of women's health-related information. We found that broad approaches, such as community advertisements in public spaces, may be less effective in recruiting audience members; rather, individualized/segmented approaches, like special feature pieces in the news media, may be more effective (especially when covering highly specialized topics). We also found that social media, while feasible and inexpensive for sharing information about an upcoming event, was not as effective in increasing face-to-face attendance at our public forums compared to newspaper and clinic advertisement.

In regards to our online activities, we found that having a clear and visually appealing home page was important for viewer engagement. It thus seems prudent for KT researchers to understand the purpose of the site and design it accordingly with these aims in mind. There is also a need to ensure SEO best practices are being followed for optimized viewer acquisition.

Regarding our development of YouTube videos, we found that short and concise videos worked well to increase viewing frequency and duration. We found the analytics tool extremely useful when designing subsequent videos as they allowed us to tailor the duration and content to fit viewer habits. In regards to our social media efforts, we found that Facebook paid ads (advertising the forum) can be helpful to engage an online audience, and that sharing online- only information can be more effective than other social media strategies. Taken together, there are some overall messages about optimizing social and physical media for knowledge translation in the domain of women's health:

a. Understanding audience behaviour is key in any medium; and

b. Planning how to reach your goals (events attended, information shared, etc) in relation to the intended/anticipated audience can help you more clearly define your strategy and tools.

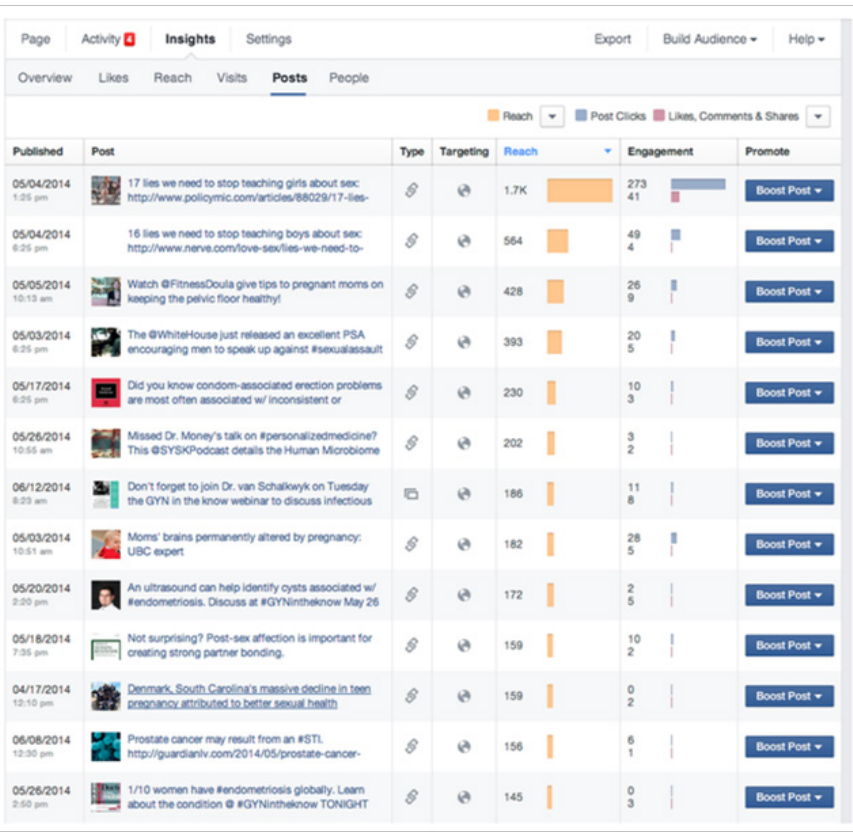

Appendix I Facebook engagement by reach 


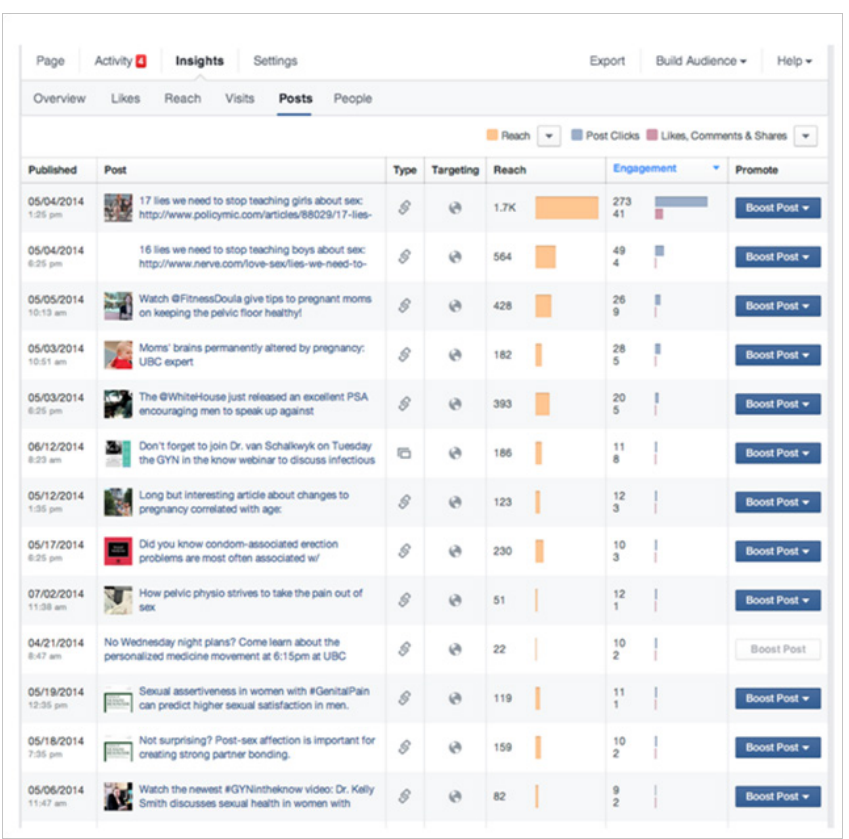

Appendix 2 Facebook engagement by post clicks

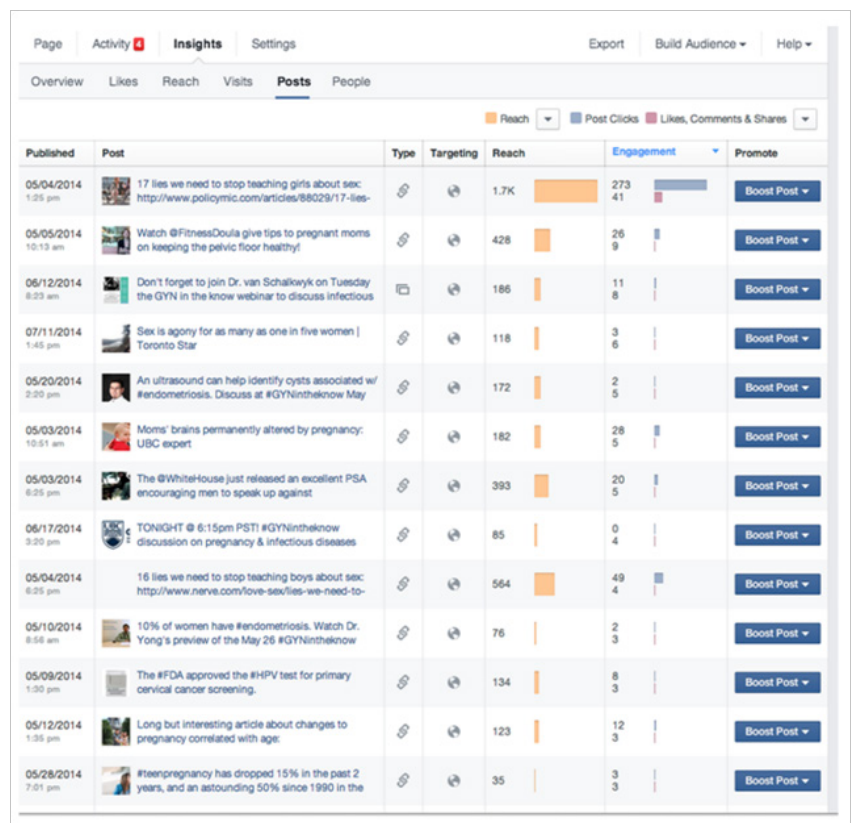

Appendix 3 Facebook engagement by likes/shares

\section{Acknowledgements}

None.

\section{Conflicts of interest}

None.

\section{References}

1. Canadian Institutes of Health Research. Knowledge translation definition. Ottawa (ON), Canada. 2014.
2. Poole N. Using consciousness-raising principles to inform modern knowledge translation practices in women's health. Can J Nurse Res. 2008;40(2):76-93

3. Straus SE, Tetroe J, Graham I. Defining knowledge translation. CMAJ. 2009;181(3-4):165-168.

4. Berwick DM. Disseminating innovations in health care. JAMA. 2003;289(15):1969-1975.

5. Landry R, Amara N, Lamari M. Utilization of social science research knowledge in Canada. Res Policy. 2001;30(2):333-349.

6. King L, Wise M, Hawe P. From research into practice in health promotion: a review of the literature on dissemination. National Centre for Health Promotion. Sydney, Australia.. 1996;p.38-42.

7. O'Grady LA, Witteman H, Wathen CN. The experiential health information processing model: supporting collaborative web-based patient education. BMC Med Inform Decis Mak. 2008;8(1):58.

8. Handfield B, Turnbull S, Bell RJ. What do obstetricians think about media influences on their patients? Aust $N$ Z J Obstet Gynaecol. 2006;46(5):379-383.

9. Bernhardt JM, Felter EM. Online pediatric information seeking among mothers of young children: results from a qualitative study using focus groups. J Med Internet Res. 2004;6(1):e7.

10. Harper CC, Blum M, de Bocanegra HT, et al. Challenges in translating evidence to practice: the provision of intrauterine contraception. Obstet Gynecol. 2008;111(6):1359-1369.

11. Tone AA, Salvador S, Finlayson, SJ, et al. The role of the fallopian tube in ovarian cancer. Clin Adv Hematol Oncol. 2012;10(5):296-306.

12. Sandoval C, Fung-Kee-Fung M, Gilks B, et al. Examining the use of salpingectomy with hysterectomy in Canada. Curr Oncol. 2013;20(3):173-175.

13. McAlpine JN, Hanley GE, Woo MM, et al. Opportunistic salpingectomy: uptake, risks, and complications of a regional initiative for ovarian cancer prevention. Am J Obstet Gynecol. 2014;210(5):471.e1-471.e11.

14. Reade CJ, Finlayson S, McAlpine J, et al. Risk-reducing salpingectomy in Canada: a survey of obstetrician-gynaecologists. J Obstet Gynaecol Can. 2013;35(7):627-634.

15. The Society of Obstetricians and Gynaecologists of Canada. Clinical practice guidelines. Ottawa (ON), Canada. 2015.

16. Davis H. Search engine optimization. O’Reilly Media, Inc, Sebastapol, CA, USA. 2006.

17. Slater JS, Finnegan Jr JR, Madigan SD. Incorporation of a successful community-based mammography intervention: dissemination beyond a community trial. Health Psychol. 2005;24(5):463-469.

18. Google Analytics Help. Google Analytics support forum. Mountain View (CA), USA.

19. Wood TJ. Exploring the role of first impressions in rater-based assessments. Adv Health Sci Educ Theory Pract. 2014;19(3):409-427.

20. Hernandez A, Resnick ML. Placement of Call to Action buttons for higher website conversion and acquisition an eye tracking study. In Proceedings of the Human Factors and Ergonomics Society Annual Meeting, SAGE Publications, Thousand Oaks: San Diego, CA, USA. 2013;57(1):1042-1046. 